\title{
MARKOV PROCESSES ON MANIFOLDS OF MAPS
}

\author{
BY PETER BAXENDALE
}

Communicated by Daniel W. Stroock, January 2, 1976

1. Introduction. In this note we describe a construction of a Markov process on a manifold of maps starting from a Gaussian measure on the space of sections of an associated vector bundle. Let $S$ be a compact metric space of finite metric dimension and $M$ a smooth complete finite dimensional Riemannian manifold. Our basic construction gives a family $\left\{v_{t}: t \geqslant 0\right\}$ of Borel probability measures on the space $C(S \times M, M)$ of continuous functions from $S \times M$ to $M$ with the compact-open topology. The multiplication $(f, g)(s, m)=$ $f(s, g(s, m))$ for $f, g \in C(S \times M, M)$ makes $C(S \times M, M)$ into a topological semigroup with identity. Then $\nu_{t} * \nu_{s}=\nu_{t+s}$ for $s, t \geqslant 0$ and the right translates of the $\nu_{t}$ give transition probabilities for a Markov process on $C(S \times M, M)$ with continuous sample paths. The left action of $C(S \times M, M)$ on $C(S, M)$ induces a Markov process on $C(S, M)$ with transition probability $\nu_{t, g}=$ image of $\nu_{t}$ under the action of $C(S \times M, M)$ on $g \in C(S, M)$.

2. Statement of results. Let $\xi$ denote the product bundle $S \times T M \rightarrow$ $S \times M$ and $C(\xi)$ the space of continuous sections of $\xi$. Given a Gaussian measure $\mu$ of mean zero on $C(\xi)$, define

$$
\begin{array}{r}
Q(s, x, t, y)=\int f(s, x) \otimes f(t, y) d \mu(f) \in T_{x} M \otimes T_{y} M \\
\text { for all } s, t \in S, x, y \in M .
\end{array}
$$

$Q$ is a reproducing kernel for the bundle $\xi$ (see Baxendale [1]) and determines $\mu$ uniquely. Let $X \in C(\xi)$.

For a closed isometric embedding of $M$ inside some Euclidean space $V$, let $h(x)$ denote the second fundamental form for $M \subset V$ at $x \in M$. Using the natural inclusion $T_{x} M \subset V$ and orthogonal projection $V \rightarrow T_{x} M$, we think of $X, Q$ and $h$ taking values in $V$ and its various tensor products.

THEOREM 1. Suppose there exists a closed isometric embedding $M \subset V$ such that (i) $h$ is bounded and uniformly Lipschitz with respect to the metric on $M$ induced from $V$.

Suppose moreover that there exist a Gaussian measure $\mu$ on $C(\xi), X \in$ $C(\xi)$ and $\alpha>0, C>0$ such that

AMS (MOS) subject classifications (1970). 58B20, 58D15, 60H10, 60J 35. 
(ii)

$$
\begin{aligned}
& \operatorname{tr}(Q(s, x, s, x)) \leqslant C, \quad \forall s, x, \\
& \begin{aligned}
& \operatorname{tr}(Q(s, x, s, x)+Q(t, y, t, y)-Q(s, x, t, y)-Q(t, y, s, x)) \\
& \leqslant C\left(d(s, t)^{2 \alpha}+|x-y|_{V}^{2}\right), \quad \forall s, x, t, y,
\end{aligned}
\end{aligned}
$$

$$
\begin{aligned}
& |X(s, x)|_{V} \leqslant C, \quad \forall s, x, \\
& |X(s, x)-X(t, y)|_{V} \leqslant C\left(d(s, t)^{\alpha}+|x-y|_{V}\right), \quad \forall s, x, t, y .
\end{aligned}
$$

Then $\mu$ and $X$ determine a family of Borel probability measures $\left\{\nu_{t}: t \geqslant 0\right\}$ on $C(S \times M, M)$ satisfying

(a) $\nu_{s} * \nu_{t}=\nu_{s+t}, \forall s, t \geqslant 0$,

(b) the $\nu_{t}$ are transition probabilities for a Markov process on $C(S \times M, M)$ with continuous sample paths.

We illustrate the dependence of the $\left\{\nu_{t}\right\}$ on $\mu$ and $X$ as follows. For $\mathbf{s}=$ $\left(s_{1}, \ldots, s_{r}\right) \in S^{r}$ and $\mathbf{x}=\left(x_{1}, \ldots, x_{r}\right) \in M^{r}$ denote by

$$
\begin{aligned}
& \rho_{\mathrm{s}, \mathrm{x}}: C(S \times M, M) \rightarrow M^{r} \\
& \sigma_{\mathrm{s}, \mathbf{x}}: C(\xi) \rightarrow T_{x_{1}} M \times \cdots \times T_{x_{r}} M,
\end{aligned}
$$

the evaluation maps at $\left(s_{1}, x_{1}\right), \ldots,\left(s_{r}, x_{r}\right)$. Let $\nu_{s, \mathbf{x}}^{t}$ be the image of $\nu_{t}$ under $\rho_{\mathbf{s}, \mathbf{x}}$, then

(i) the $\nu_{\mathrm{s}, \mathbf{x}}^{t}$ for all $\mathbf{s}, \mathbf{x}$ determine $\nu_{t}$,

(ii) the $\nu_{\mathbf{s}, \mathbf{x}}^{t}$ for fixed $\mathbf{s}$ are the transition probabilities for a Markov process on $M^{r}$ with continuous sample paths.

THEOREM 2. The Markov process corresponding $\left\{\nu_{\mathbf{s}, \mathbf{x}}^{t}: t \geqslant 0, \mathbf{x} \in M^{r}\right\}$ has infinitesimal generator $A_{\mathbf{s}}$, where

$$
\left(A_{\mathbf{s}} g\right)(\mathbf{x})=\frac{1}{2} \int\left(\nabla^{2} g\right)(\mathbf{x})\left(\sigma_{\mathbf{s}, \mathbf{x}}(h), \sigma_{\mathbf{s}, \mathbf{x}}(h)\right) d \mu(h)+(\nabla g)(\mathbf{x})\left(\sigma_{\mathbf{s}, \mathbf{x}}(X)\right)
$$

where $\nabla$ is covariant differentiation with respect to the product Riemannian structure on $M^{r}$.

3. The construction. For each $\mathbf{s}, \mathbf{x}$ and $t>0$, we construct a measure $\nu_{\mathrm{s}, \mathbf{x}}^{t}$ on $M^{r}$ as follows. Using the embedding $M \subset V$ and choosing suitable extensions, we construct a Wiener process $W_{t}$ in $C(S \times V, V)$ (see Gross [2]) and $\widetilde{X} \in C(S \times V, V)$. Define

$$
Y(s, x)=\frac{1}{2} \int_{C(\xi)} h(x)(g(s, x), g(s, x)) d \mu(g) \in T_{x}^{\perp} M \quad \text { for } x \in M,
$$

and extend to $\tilde{Y} \in C(S \times V, V)$. Consider the stochastic differential equation in $V^{r}$ 


$$
\left.\begin{array}{rl}
d \eta_{i}(t) & =(\tilde{X}+\tilde{Y})\left(s_{i}, \eta_{i}(t)\right) d t+d W(t)\left(s_{i}, \eta_{i}(t)\right) \\
\eta_{i}(0) & =x_{i}
\end{array}\right\} i=1, \ldots, r .
$$

The choice of $\widetilde{Y}$ ensures that if $x_{i} \in M$, then $\eta_{i}(t) \in M$ for all $t>0$ with probability one. The conditions (i), (ii) and (iii), plus care in choosing extensions, imply that the equation has a solution for all $t>0$, that the solution is continuous with probability one and has finite moments of all orders. We define $\nu_{\mathbf{s}, \mathbf{x}}^{t}$ to be the distribution of $\left(\eta_{1}(t), \ldots, \eta_{r}(t)\right) \in M^{r}$. The existence of the $\left\{\nu_{t}\right\}$ follows from the Daniell-Kolmogorov construction and an estimate on the moments of solutions of the stochastic differential equation.

4. Examples. Suppose $S$ and $M$ are compact Riemannian manifolds and $p>1 / 2 \operatorname{dim} S, q>1 / 2 \operatorname{dim} M+1$. Then $L_{p}^{2}(S) \otimes L_{q}^{2}(T M) \subset C(\xi)$ is radonifying, and the Wiener measure $\mu$ satisfies the conditions of Theorem 1. Take $X=0$. Each pair $(p, q)$ in the range above yields a different family $\left\{v_{t}\right\}$ of measures on $C(S \times M, M)$.

The condition that $M$ be compact may be replaced by completeness together with certain curvature conditions.

Notice that the case $M=\mathbf{R}^{n}$ yields Gaussian measures. Also $S=$ point and suitable choice of $\mu$ gives Brownian motion on $M$ under the sole condition that there exists a closed isometric embedding with $\|h(x)\| \leqslant C\left(1+d\left(x, x_{0}\right)\right)$ for some $C>0$ and $x_{0} \in M$.

\section{REFERENCES}

1. P. Baxendale, Gaussian measures on function spaces, Amer. J. Math. (to appear).

2. L. Gross, Potential theory on Hilbert space, J. Functional Analysis 1 (1967), 123-181. MR 37 \#3331.

DEPARTMENT OF MATHEMATICS, KING'S COLLEGE, ABERDEEN, SCOTLAND 